\title{
ANALYSIS AND STUDY ON 47 CASES OF ADVERSE REACTIONS OF CHINESE MEDICINE INJECTION
}

\author{
Xiaoyu Zhang $^{1 *}$, Dechun Sang ${ }^{1,}$ Zhiqiang Zhang ${ }^{2,}$ Shuangyan Kong ${ }^{3}$ \\ ${ }^{1}$ Department of General Rehabilitation, China Rehabilitation Research Center, Capital Medical University School of rehabilitation medicine, \\ Beijing 100068, China. ${ }^{2}$ Institute of Basic Research in Clinical Medicine, China Academy of Chinese Medical Sciences, Beijing 100700, China. \\ ${ }^{3}$ Department of Neurology, Aviation General Hospital, Beijing 100012, China. \\ *E-mail: nmbrbd@163.com
}

\begin{abstract}
Background: Along with efficacy, Chinese medicine is increasingly being known to people, Chinese medicine and its preparations are increasingly widespread in clinical use. People generally believe that Chinese medicine has few side effects and is safe, especially Chinese medicine injections. Due to the direct injection in blood, rapid onset and good efficacy, they are welcomed by people. However, with increased use, adverse reactions are increasing, even causing serious consequences. The objective is to learn about the characteristics and laws of the adverse effects of Chinese medicine injections, provide references for the clinical safe drug use, and reduce the incidence of adverse reactions.
\end{abstract}

Materials and Methods: The method was established to analyze the data of 47 cases of adverse reactions caused by Chinese medicine injections in our hospital from the year, 2009 to 2010.

Results: The organs / systems involved in the 47 cases of adverse reactions are primarily skin and its accessories and secondly systemic damage, involving a total of eight varieties of drugs.

Conclusion: The adverse reactions of Chinese medicine injections are mostly in Chinese patent drugs, which should be paid attention to, to find out the problems and laws, use the drugs rationally, and reduce the incidence of the adverse reactions.

Key words: Chinese medicine; Injection; Adverse reactions

\section{Introduction}

Chinese medicine injections are sterile solutions made from the effective substances extracted from medicinal materials with modern scientific techniques and methods. Along with efficacy, Chinese medicine is increasingly being known to people, Chinese medicine and its preparations are increasingly widespread in clinical use. In China, people generally believe that Chinese medicine has few side effects and is safe, especially Chinese medicine injections. Due to the direct injection in blood, rapid onset and good efficacy, they are welcomed by people (Li et al., 2010a). However, with increased use, adverse reactions are increasing, even causing serious consequences. This paper makes the statistics and analysis on the reported adverse reactions caused by Chinese medicine injections collected in our hospital in 2009 2010, to provide references for clinical use (Zhang et al., 2006).

\section{Data source}

47 cases of adverse reactions caused by Chinese medicine injections collected in outpatient and inpatient departments in our hospital from 2009 to Dec. 2010.

\section{Methods}

Summary and analysis are made on the 47 cases of adverse reactions caused by Chinese medicine injections according to gender, ages, family history of allergies, drug classification and clinical manifestations of adverse reactions.

\section{Results}

Distribution of ages and gender of the patients with adverse reactions

The 47 patients are 19 males (40.43\%) and 28 females (59.57\%), with the male to female ratio of 1:1.47. The incidence of adverse reactions caused by Chinese medicine injections in female is higher than that in male. In the 47 cases of adverse reactions caused by Chinese medicine injections, the youngest patient is 2 years old, and the oldest is 83 years old. Specific age distribution is shown in Table 1. 
http://dx.doi.org/10.4314/ajtcam.v11i2.23

Table 1: Distribution of ages and gender of the patients with adverse reactions

\begin{tabular}{ccccc}
\hline Age & Male & Female & $\mathrm{n}$ & Proportion (\%) \\
\hline $0-19$ & 0 & 2 & 2 & 4.26 \\
$20-39$ & 1 & 4 & 5 & 10.64 \\
$40-60$ & 5 & 10 & 15 & 31.91 \\
$>60$ & 13 & 12 & 25 & 53.19 \\
Total & 19 & 28 & 47 & 100.00 \\
\hline
\end{tabular}

\section{Varieties of drugs causing adverse reactions and number of cases}

47 cases of adverse reactions involve 8 drug varieties, as shown in Table 2.

Table 2: Varieties and proportions of 8 Chinese medicine injections causing adverse reactions

\begin{tabular}{lcc}
\hline Drug name & $\mathrm{n}$ & Proportion (\%) \\
\hline Acanthopanax Injection & 18 & 38.30 \\
Shuxuening Injection & 10 & 21.28 \\
Tanreqing Injection & 6 & 12.77 \\
Xingnaojing Injection & 4 & 8.51 \\
Shenmai Injection & 4 & 8.51 \\
Shuanghuanglian Injection & 3 & 6.37 \\
Xuesaitong Injection & 1 & 2.13 \\
Safflower Injection & 1 & 2.13 \\
Total & 47 & 100.00 \\
\hline
\end{tabular}

\section{Clinical manifestations of adverse reactions}

The reports of 47 cases involve the damages to a total of 8 organs / systems. In which, skin and its accessories are involved most, manifested as pruritus, rash, etc, because skin manifestations are easy to observe and not easily confused, detailed in Table 3.

Table 3: Varieties and clinical manifestations of adverse reactions caused by Chinese medicine injections

\begin{tabular}{llll}
\hline Involved system / organ & Clinical manifestations & $\mathrm{n}$ & Proportion $(\%)$ \\
\hline Skin and its accessories & Rash, pruritus and various dermatitis & 28 & 59.57 \\
Systemic damage & Fever, chill, allergic shock, general weakness and sweating & 17 & 36.17 \\
Cardiovascular system & Palpitation, chest tightness, abnormal blood pressure and pale face & 12 & 25.53 \\
Digestive system & Nausea, vomiting, abdominal pain, appetite decrease and constipation & 6 & 12.77 \\
Nervous system & Dizziness, headache, flush, confusion and myasthenia & Coughing, expiratory dyspnea, asthma and shortness of breath & 5 \\
Respiratory system & Frequent urination, urgent urination and blood urine & 5 & 10.64 \\
Urinary system & Pain and swelling & 2 & 4.25 \\
Effect-site damage & n & 4.25 & 2 \\
\hline
\end{tabular}

Family history of allergies of the 47 patients with adverse reactions

Table 4: Family history of allergies

\begin{tabular}{ccc}
\hline History of allergies & $\mathrm{n}$ & Proportion $(\%)$ \\
\hline Yes & 8 & 17.02 \\
None & 23 & 48.94 \\
Unclear & 16 & 34.04 \\
Total & 47 & 100.00 \\
\hline
\end{tabular}

$\mathrm{n}=$ number of patients 
http://dx.doi.org/10.4314/ajtcam.v11i2.23

\section{Discussion}

The statistical results show that the patients are 19 males and 28 females, with the male to female ratio of 1:1.47. The incidence in female is higher than that in male. The incidence of adverse reactions in patients with age $>60$ years is higher, mainly because they are older, easy to incur many diseases and willing to take many drugs at same time, and have decreased physiological function and drug metabolism, therefore prone to adverse reaction.

It can be seen from Table 3 that the adverse reactions caused by Chinese medicine injections involve many human organs, and some present multi-organ reactions. In cases of allergic reactions, the drug-induced allergic reactions account for about 10\% (Suzanne et al., 2007). The adverse reactions caused by Acanthopanax injection are mostly typical I-type allergic reactions, mainly manifested as allergic dermatitis and gastroenteritis (Wang et al., 2009; Li et al., 2010b). The adverse reactions caused by Shuxuening injection are mostly damages to skin and its accessories, single-system damages are rare, while multi-system damages are many (Huang, 2009), such as digestive and respiratory system damages, and the severe cases can incur allergic shock (Lv et al., 2010). The adverse reactions caused by Tanreqing injection involve primarily skin and its accessories and secondly peripheral vascular and gastrointestinal reactions, etc (Peng, 2011). The adverse reactions caused by Xingnaoning injection are mainly allergic reactions, such as rash and allergic shock, manifested as flush, chest tightness, dyspnea and other symptoms (Dai et al., 2009). Shenmai injection is widely used in clinical treatment of pulmonary heart disease, cardiomyopathy, myocardial infarction and so on, its adverse reactions are mainly allergies, as well as cardiovascular system damages, mostly manifested as chest tightness, palpitation and other symptoms, and respiratory system, nervous system and digestive system are also involved (Li, 2010; Li et al., 2010a). Shuanghuanglian injection is mainly composed of honeysuckle, scutellaria and fructus forsythiae, with the effects of clearing heat and detoxicating, and its adverse reactions are primarily allergic reactions and secondly digestive system reactions, similar to the related reports (Larrey et al., 1998; Gao et al., 2010). Xuesaitong injection mainly consists of total ginsenoside Rb1, ginsenoside Rg1 and notoginsenoside R1 and is clinically widely used in cerebral embolism, coronary heart disease and other diseases, and its adverse reactions mainly involve circulatory system, skin, respiratory system and nervous systems, mainly manifested as pruritus, blood pressure decrease, dyspnea and other symptoms (Lei et al., 2010). Safflower injection is extracted from the Chinese medicine safflower carthamus and mainly used for clinical treatment of coronary heart disease and other diseases, and its adverse reactions are mainly manifested as pale face, chest tightness, shortness of breath, pruritus, fever and other symptoms (Kang, 2010).

In the 47 cases of adverse reactions, 8 cases have history of allergies, accounting for $17.02 \%, 23$ cases have no history of allergies, accounting for $48.94 \%$, and 16 cases are unclear, accounting for $34.04 \%$. The result suggests that before drug use, doctors should ask if there is history of allergies, or the incidence of adverse reactions will be undoubtedly increased.

Different individuals and different gender and ages have different sensitivity to adverse drug reactions, of course, family history of allergies is also critical, and few adverse reactions are related with allergic constitution, so clinical medical staff should pay great attention to the patient's history of allergies. Of course, the factors of Chinese medicine injections themselves are also very important; for example, chlorogenic acid and isochlorogenic acid contained in Shuanghuanglian injection are highly sensitizing antigens and can induce high sensitization after entering into the body as antigens, being one of the main causes of allergic reactions (Zhang et al., 2006). Other components are easy to be mixed in Chinese medicine injections during preparation, and storage and use are likely to cause adverse reactions. Furthermore, the drug interaction is also a cause of adverse reactions, and when many drugs are used at the same time, the incidence of adverse reactions increases. Therefore, medical staff should pay attention to adverse reactions and handle and report them in time when finding, and storage and use of drugs should also be paid attention to.

\section{References}

1. Dai, F. W., Li, M., Lei. G. Y., and Lei, Z. B. (2009). Analysis on 16 Cases of Adverse Reactions Caused by Xingnaojing Injection. Strait Pharmaceutical Journal, 21(8): 191-193.

2. Gao, Y. Q., Su, D., and Zeng, C. Y. (2010). Literature Analysis on 58 Cases of Adverse Reactions Caused by Shuanghuanglian Injection. Chinese Journal of Clinical Rational Drug Use, 3(4): 71-72.

3. Huang, B. (2009). Literature Analysis on Adverse Reactions Caused by Shuxuening Injection. Evaluation and Analysis of Drug-use in Hospitals of China, 9(12): 944-945.

4. Kang, J. (2010). Analysis on 60 Cases of Adverse Reactions Caused by Safflower Injection. China Medical News, 29(11): $1520-1521$. 
http://dx.doi.org/10.4314/ajtcam.v11i2.23

5. Larrey, D., Erlinger, S., and Krishnasuamy, K. (1998). Hepatic Dysfunction in Undernourished Patients Receiving in Soniazid and Rifampicin. Baillieres Clinical Gast roenterology, 23(4): 423-431.

6. Lei, G. Y., and Lei, Z. B. (2010). Retrospective Analysis on 36 Cases of Adverse Reactions Caused by Xuesaitong Injection. Chinese Traditional Patent Medicine, 32(5): 851-853.

7. Li, S. H., Li, X., and Wei, X. B. (2010a). Literature Analysis on 91 Cases of Adverse Reactions Caused by Acanthopanax Injection. Chinese Journal of Modern Drug Application, 4(5): 151-152.

8. Li, Y. R., and Pan, T. X. (2010b). Literature Analysis on 170 Cases of Adverse Reactions Caused by Shenmai Injection. Chinese Journal of Pharmacovigilance, 7(10): 623-625.

9. Li, Y. L. (2010). Literature Analysis on 167 Cases of Adverse Reactions Caused by Shenmai Injection. Guangming Journal of Chinese Medicine, 25(2): 126-128.

10. Lv, Y. Q., Li, C. X., and Diao, J. L. (2010). Analysis on 17 Cases of Adverse Reactions Caused by Shuxuening Injection. Journal of Diseases Monitor and Control, 4(6): 370-371.

11. Peng, J. (2011). Analysis on 42 Cases of Adverse Reactions Caused by Tanreqing Injection. Pharmaceutical Care Research, 11(4): $317-319$.

12. Suzanne, L. W., Aimee, A. D. A, and Tamar, R. K. (2007). Population-based Drug-Related Anaphylaxis in Children and Adolescents Captured by South Carolina Emergency Room Hospital Discharge Database (SCERHDD) (2000-2002). Pharmaceutical Drug Safety, 16 (2): 1255-1267.

13. Wang, J., and Liao, L. (2009). Investigation and Analysis on 158 Cases of Adverse Reactions Caused by Acanthopanax Injection. Journal of Traditional Chinese Medicine, 6(21): 91-93.

14. Zhang, H. X., Chen, J. Y., and Song, C. (2006). Analysis on 3414 Cases of Adverse Reactions caused by Chinese Medicine Injections. Chinese Journal of Pharmacovigilance, 3(4): 232-236. 